\title{
PRODUÇÃO CIENTÍFICA E FORMAÇÃO DE RECURSOS HUMANOS NA ÁREA DE BIOQUÍMICA EM INSTITUIÇÕES FEDERAIS DO RIO GRANDE DO SUL: FOMENTO ESTADUAL
}

\author{
Luciana Calabró Berti*, Diogo L. Oliveira, Diogo O. Souza e Susana T. Wofchuk \\ Departamento de Bioquímica, Instituto de Ciências Básicas da Saúde, Universidade Federal do Rio Grande do Sul, Rua Ramiro \\ Barcellos, 2600 - Anexo, 90035-003 Porto Alegre, RS - Brasil
}

Recebido em 23/4/09; aceito em 16/9/09; publicado na web em 21/1/10

\begin{abstract}
SCIENTIFIC PRODUCTION AND THE FORMATION OF HUMAN RESOURCES IN THE FIELD OF BIOCHEMISTRY IN FEDERAL INSTITUTIONS IN RIO GRANDE DO SUL: STATE FINANCIAL AID. Brazilian scientific production in Biochemistry is growing impressively fast, and Rio Grande do Sul is outstanding in that context. This study aims to outline the state's scientific research profile, given its prominent position in the national scenario. Hence, researchers, laboratories, development of human resources and investments by Foundation for the Support to Research in the State of Rio Grande do Sul (FAPERGS) were identified and mapped. We observed that amount of financial support by FAPERGS decreases in last years. Therefore, there is the necessity to reestablish some of FAPERGS's programs, as well as to increase financial support to Biochemistry departments.
\end{abstract}

Keywords: scientific production; FAPERGS; biochemistry.

\section{INTRODUÇÃO}

\section{Produção científica e divulgação}

A produção científica brasileira medida em publicações tem crescido em contribuição à produção internacional. O Brasil foi responsável por cerca de $1,5 \%$ da publicação científica mundial no período 1999-2003 (passando da $23^{\mathrm{a}}$ posição para $17^{\mathrm{a}}$ ), apresentando o melhor ranking da América Latina. ${ }^{1}$

A ciência no Brasil foi institucionalizada no século XIX. ${ }^{2}$ A necessidade e a aspiração crescentes de desenvolver ciência e treinar novos pesquisadores levaram a mudanças estruturais responsáveis pelo crescimento da ciência no Brasil. ${ }^{3}$

Assim, na última década, houve um crescimento importante na produção científica brasileira, da qual em torno de $90 \%$ foi em instituições públicas com programas de Pós-Graduação (PG). Portanto, podemos dizer que a ciência está localizada em algumas poucas instituições, ${ }^{3}$ principalmente na universidade pública. ${ }^{4} \mathrm{O}$ programa nacional de formação de recursos humanos para ciência, principalmente a partir de 1990, contribuiu em grande parte para o aumento de produtividade. ${ }^{1}$ Os cientistas consideram de grande importância a participação de alunos para sua produção: "a dupla orientadorestudante pode ser o fator mais forte para desencadear produtividade". Portanto, há uma relação positiva entre incremento da produtividade científica e expansão dos cursos de PG e consequente aumento no número de estudantes, mesmo em períodos em que o investimento de recursos é baixo. ${ }^{5}$

No que se refere à divulgação científica, publicar os resultados de suas pesquisas é um compromisso que os cientistas são compelidos a cumprir. ${ }^{6} \mathrm{O}$ avanço do conhecimento produzido pelos pesquisadores tem de ser transformado em informação acessível para a comunidade científica. A pesquisa é desenvolvida em um contexto de troca: "A publicação dos resultados de pesquisa tem três objetivos: divulgar descobertas científicas, salvaguardar a propriedade intelectual e alcançar a fama". ${ }^{7}$

Um documento científico é, ao mesmo tempo, mais e menos que um conceito ou um dado ou uma hipótese. Se o documento é a

\footnotetext{
*e-mail: luciana.berti@ufrgs.br
}

expressão de uma pessoa ou de um grupo trabalhando em uma frente de pesquisa, podemos dizer alguma coisa sobre as relações entre as pessoas a partir dos próprios documentos. ${ }^{8}$

Na comunidade acadêmica existe uma forte pressão para publicar, uma vez que a progressão na carreira nas universidades e institutos de pesquisa tem como uma das bases de avaliação a produção científica. ${ }^{9}$

A produção do conhecimento gerado nas universidades é divulgada em diferentes canais que variam de área para área, ou mesmo de pesquisa para pesquisa, sendo o documento formal o meio mais reconhecido para dar maior visibilidade aos estudos e pesquisas realizados, transformando-os em uma força motriz, na medida em que são divulgados, impulsionando o desenvolvimento intelectual e realimentando o ciclo da geração do conhecimento. ${ }^{9}$

Muitas publicações são feitas em revistas científicas locais e não indexadas orientadas para público local, cujo valor parece não ser claro. Muitos cientistas brasileiros ainda preferem publicar em revistas nacionais ou da América Latina. ${ }^{1}$ Esta estratégia vem em detrimento da visibilidade. Por outro lado, ${ }^{10}$ referem que há uma tendência de diminuir estas publicações e aumentar aquelas com visibilidade internacional. Isto pode ser explicado pelo aumento de interações entre grupos nacionais e internacionais, ocasionando aumento em copublicações científicas, o que provavelmente ajudou a consolidação da ciência brasileira. ${ }^{11}$

\section{Indicadores}

Atualmente, os indicadores da atividade científica estão no centro dos debates sobre a relação do avanço da ciência e da tecnologia com o progresso econômico e social. Estratégias de políticas públicas para o setor seriam inconcebíveis sem a possibilidade de se contar com indicadores objetivos de desempenho científico. Os indicadores, eles mesmos, se sofisticam de modo a dar conta da complexidade dos processos de pesquisa e seus resultados. ${ }^{12}$ Se por muito tempo o foco das avaliações permaneceu orientado para medir os parâmetros como verbas e pessoal de pesquisa e desenvolvimento (P\&D), crescentemente o interesse está se voltando para os indicadores de resultados. ${ }^{13}$ Em tudo o que se refere à ciência, os indicadores cienciométricos tornaram-se essenciais.

Vários procedimentos estão sendo adotados para acompanhar 
o desenvolvimento da pós-graduação, ${ }^{14-16}$ avaliar os resultados em áreas específicas ${ }^{17-19}$ e questionar diferentes aspectos da produção científica brasileira. $^{20,21}$

Fonseca e colaboradores ${ }^{22}$ referem que o desempenho científico é um tema controverso, mas que alta produtividade está relacionada à excelência e leva a contribuições notáveis. Mesmo que a produtividade dos pesquisadores individualmente tenha picos (flutuações temporárias) ao longo dos anos, o conjunto de pesquisadores em uma instituição como um todo representa uma média importante, caracterizando crescimento ou não. Quantidade de publicações é o mais forte indicador de competência. ${ }^{23,24}$

\section{Apoio à pesquisa}

O acompanhamento da produção científica e tecnológica é feito pelos órgãos de fomento, que o utilizam para estabelecer suas políticas de financiamento. Em todos os programas de fomento e financiamento das atividades de pesquisa, públicos ou privados, é importante avaliar os resultados, ou seja, os ganhos da pesquisa em função do recurso investido. ${ }^{25}$

O aumento no investimento em Pós-graduação é a principal contribuição para produção científica, assim como é importante também a institucionalização da ciência, investimento nos locais com vocação para produção científica e treinamento de cientistas. ${ }^{22}$ Desta forma, apoios governamentais são fundamentais, ${ }^{25}$ mesmo que em períodos de crise econômica, investimento seletivo em recursos humanos pode levar ao aumento da produtividade científica. ${ }^{26}$

\section{Bioquímica no Brasil}

A área de Bioquímica tem uma contribuição importante na produção de conhecimento no Brasil.

A literatura brasileira registra raros trabalhos abordando a evolução da Bioquímica no país. ${ }^{27}$ Referindo-se especificamente à Pós-Graduação, ${ }^{28}$ acerca da evolução da Bioquímica no Brasil anteriormente a 1965, os dados estatísticos divulgados pela CAPES são as fontes de maior utilidade para se traçar uma panorâmica do desenvolvimento dos cursos de Pós-graduação dessa área no país a partir dos anos 60 .

No Brasil, anteriormente ao século XX, os únicos trabalhos científicos em Biologia dignos de menção foram os desenvolvidos pelo naturalista Fritz Muller e pelo fisiologista João Batista de Lacerda. ${ }^{29}$ Ainda que no período entre 1808 e 1928 tenham aparecido no Brasil “...escolas ou organizações superiores onde a Bioquímica poderia ter sido cultivada ... somente em 1930 apareceram no país os precursores do desenvolvimento ininterrupto da Bioquímica". ${ }^{30}$

A atividade de investigação científica em Bioquímica foi realizada inicialmente em algumas poucas instituições, geralmente localizadas no eixo Rio (Instituto Manguinhos, Instituto de Biofísica da Universidade do Brasil) - São Paulo (Instituto Butantan, Instituto Biológico, Departamento de Química da USP, Departamento de Química Fisiológica da Faculdade de Medicina da USP) - Belo Horizonte (Faculdade de Medicina da Universidade de Minas Gerais).

Há também registros de atividades importantes na Faculdade de Medicina da Universidade do Paraná. Em algumas dessas instituições, a atividade de Pós-graduação começou a florescer. Teses de Doutorado e de Livre Docência foram defendidas na Faculdade de Medicina da Universidade de Minas Gerais (a partir dos anos 40, orientadas por Baeta Vianna), na Faculdade de Medicina da Universidade do Paraná (7 teses de Doutorado e 2 de Livre Docência, nas décadas de 40 e 50) e no Instituto de Biofísica da Universidade do Brasil (um total de 13 teses, entre 1946 e 1959).

Foi no Departamento de Química da USP que a Pós-graduação em Bioquímica se instalou com maior vigor, a partir dos anos $40 .{ }^{31} \mathrm{~A}$ criação da Universidade de São Paulo, ocorrida em 1934, é um dos mais importantes marcos do ensino superior brasileiro. Pela primeira vez o país via nascer uma instituição de ensino superior que associava ensino e pesquisa e que se orientava para o progresso da ciência.

No final da década de 60, quando a Pós-graduação foi oficialmente instituída no país, existiam cinco cursos de pós-graduação em Bioquímica, regularmente organizados, ministrados pelas seguintes universidades federais: de Minas Gerais (mestrado = M e doutorado = D), de Pernambuco (M), do Paraná (M e D), do Rio Grande do Sul (M) e do Rio de Janeiro (M e D). Nos dois primeiros anos da década seguinte, quatro novos cursos foram instituídos oficialmente, sendo por eles responsáveis as seguintes instituições: Escola Paulista de Medicina (M e D), Federal do Ceará (M), USP - São Paulo (M e D) e USP - Ribeirão Preto (M e D).

Nos anos 80, foram criados mais três cursos na área: Fundação Oswaldo Cruz (M e D), UFRJ - Química Biológica (M e D) e Universidade Estadual de Campinas (inicialmente apenas o mestrado, em 1985, ampliando-se para o doutorado, em 1990). Finalmente, em 1992, o curso da UFRGS instituiu também o doutorado e quatro anos depois foi criado o curso de mestrado na Universidade Federal do Rio Grande do Norte.

No ano de 2001, existiam 13 programas de pós-graduação em Bioquímica no Brasil, sendo que a maioria deles já se concentrava em dois estados da Federação - Rio de Janeiro e São Paulo.

Atualmente, existem 16 Programas Pós - Graduação em Bioquímica no Brasil, sendo, 14 de Mestrado e Doutorado, 1 apenas de Mestrado e 1 de Mestrado Profissionalizante, perfazendo um total de 30 cursos. ${ }^{32}$

\section{Fomento à pesquisa no Estado do Rio Grande do Sul: área Bioquímica}

A FAPERGS - Fundação de Amparo à Pesquisa do Estado do Rio Grande do Sul - é fonte de fomento público. A entidade é um órgão governamental vinculado à Secretaria Estadual da Ciência e Tecnologia - RS e responsável pela alocação de recursos para o desenvolvimento da atividade científica no Estado. A Fundação existe há 44 anos e, por força da Constituição Estadual de 1990 (artigo 236), teria como receita $1,5 \%$ do orçamento do Estado em cada exercício. A média, entretanto, no período considerado, não chega a $0,3 \%$. Além disso, a Fundação tem a finalidade de captar recursos junto a órgãos de fomento federais e internacionais.

Dos programas de fomento mantidos pela FAPERGS, procurou-se destacar, neste estudo, as iniciativas de apoio à área de Bioquímica.

A condição de vanguarda do Rio Grande do Sul (RS) nessa área de pesquisa científica despertou nosso interesse por conhecer seu perfil e realizar uma busca por informações que permitam mapear pesquisas, pesquisadores, laboratórios, formação de recursos humanos e investimentos.

A produção científica brasileira na área de Bioquímica é admiravelmente crescente e, nesse contexto, o RS é um dos centros que se sobressai. Em razão disto, a área de Bioquímica será o alvo deste estudo que buscará apontar setores e/ou departamentos de Bioquímica que mereceriam receber atenção e fomento, de forma a contribuir para o conceito positivo do qual goza o RS perante a comunidade científica nacional e internacional.

\section{OBJETIVOS}

\section{Objetivo geral}

Analisar a produção científica e formação de recursos humanos em Bioquímica nos grupos de IES públicas do Rio Grande do Sul e o impacto do fomento estadual sobre este desempenho. 


\section{Objetivos específicos}

Coletar informações sobre o perfil e atividades científicas da comunidade bioquímica do RS presente nas Universidades Federais, estudando a produtividade (artigos publicados e orientações de Pós-Graduação) desta comunidade; levantar os benefícios concedidos, através de investimentos financeiros provenientes do Governo do Rio Grande do Sul, às instituições públicas federais de pesquisa na área da bioquímica; analisar os valores destinados a cada Departamento/Setor de Bioquímica nas Universidades Federais do RS, frente à produção científica, quantificada em artigos publicados em periódicos e formação de recursos humanos na forma de orientações de mestrado e doutorado concluídas, para estabelecer relação entre os valores aplicados anualmente e a conseqüente produção científica.

\section{METODOLOGIA}

Este item tem por finalidade apresentar passo a passo os procedimentos metodológicos adotados no desenvolvimento da pesquisa.

"O processo formal e sistemático de desenvolvimento do método científico. O objetivo fundamental da pesquisa é descobrir respostas para problemas mediante o emprego de procedimentos científicos". 33

\section{Bioquímica no RS - Instituições Federais}

A investigação ocorreu através de etapas distintas. Inicialmente, identificaram-se as cinco Universidades Federais, sediadas no Rio Grande do Sul, que possuem Departamentos de Bioquímica ou Setores de Bioquímica ligados a Departamentos de Fisiologia ou Química e que realizam comprovadamente ensino e pesquisa em Bioquímica. Tornaram-se alvo de estudo a UFRGS - Universidade Federal do Rio Grande do Sul, a UFCSPA - Universidade Federal de Ciências da Saúde de Porto Alegre, a UFSM - Universidade Federal de Santa Maria, a UFPel - Universidade Federal de Pelotas e a FURG - Fundação Universidade Federal de Rio Grande. Optou-se por fazer este estudo avaliando o período de 1999 a 2007.

Os setores ou departamentos foram contatados, por meio de telefonemas e correio eletrônico. As informações foram obtidas através de responsáveis ou representantes dos departamentos/setores aos quais se solicitaram as listagens de professores em atividade no período de 1999 a 2007.

\section{Lista de professores}

De cada setor ou departamento, levantaram-se os nomes dos professores em atuação no período 1999-2007.

\section{Análise do CV}

De posse dessas listas, extraiu-se do web site do CNPq, o currículo modelo Lattes dos professores constantes do estudo, a partir dos quais foram analisados dados no período a que se refere o estudo. Levantou-se também, a produção geral individual para análise histórica da atividade científica dos professores e/ou pesquisadores.

Os dados extraídos foram aqueles acerca da titulação - graduação, mestrado, doutorado, pós-doutorado - além da sua produção científica e da contribuição na formação de recursos humanos, medida pelo número de mestres/doutores formados. Os dados referentes à produção científica coletada (artigos publicados) foram separados ano a ano por professor e sobrepostos, para que não fossem repetidos.

\section{Análise do perfil}

O passo seguinte foi categorizar, a partir dos currículos Lattes de cada professor, informações relevantes como sexo, formação de graduação e pós-graduação - destacando as instituições de ensino superior onde foi feita esta formação, bem como os orientadores de mestrado, doutorado e pós-doutorado.

Considerou-se para análise o tempo de atuação como professor e/ou pesquisador no respectivo departamento/setor dentro do período selecionado de 1999-2007.

\section{Produção científica}

Para avaliação da produção científica, analisou-se a totalidade de artigos completos publicados em periódicos científicos pelos professores/pesquisadores enquanto estiveram vinculados aos Departamentos/Setores de Bioquímica. Verificaram-se, ainda, todas as orientações de mestrado e doutorado concluídas neste período, em qualquer Programa de Pós-Graduação aos quais os professores estivessem vinculados.

Os dados de publicação total dos Departamentos/Setores de Bioquímica analisados foram levantados para identificação de dados da produção daquele setor no período analisado. Após, foi feita a análise, ano a ano, da produção referente ao período em estudo (1999-2007). Considerou-se como produção científica os artigos publicados em revistas não indexadas e indexadas ao banco de dados do Institute for Scientific Information (ISI).

\section{Levantamento de auxílios e bolsas da FAPERGS}

Através do sistema de Auxílios e Bolsas da FAPERGS, selecionaram-se os mesmos professores e se obtiveram os valores das bolsas, dos auxílios individuais, institucionais e os editais concedidos no período estudado.

Esta etapa concentrou-se na seleção de todos os professores dos departamentos/setores de Bioquímica de Universidades Federais do Rio Grande do Sul no período de 1999 a 2007, com a subsequente avaliação das modalidades de auxílios recebidos, sejam individuais ou institucionais. Observou-se o número de bolsas concedidas visando a formação de recursos humanos e a quantidade de auxílios objetivando fomentar pesquisas e promover o intercâmbio científico e tecnológico. Após, computou-se o valor total investido nos pesquisadores e Departamentos/Setores por meio dos auxílios.

\section{RESULTADOS E DISCUSSÃO}

A análise dos dados foi iniciada caracterizando-se o perfil dos professores pertencentes a Departamentos e Setores de Bioquímica das Instituições Federais de Ensino Superior no RS, em atividade no período de 1999-2007. Conforme se observa na Tabela 1, a UFRGS possui o maior número de professores atuando em Departamento de Bioquímica, em relação às demais instituições pesquisadas, com leve predominância do sexo feminino, o que também ocorre na UFSM. Na UFCSPA, as mulheres formam a totalidade. Já na FURG, há 100\% de homens, enquanto na UFPel, a maioria também é masculina.

Com relação à formação graduada dos pesquisadores, na UFRGS e na UFSM percebe-se que há mais farmacêuticos, que atingem quase a metade dos professores. Na UFRGS, a segunda maior incidência é de médicos e na UFSM de biólogos. Também é significativa a presença de biólogos na UFRGS e de químicos na UFSM. A situação se inverte com maior número de biólogos na UFCSPA e na FURG. Somente na UFPel existe uma maioria formada por agrônomos e médicos veterinários (na Tabela 1 descritos como "outros") e também químicos. 
Tabela 1. Perfil dos Professores de Bioquímica nas Instituições Federais do RS no período de 1999-2007

\begin{tabular}{|c|c|c|c|c|c|c|c|c|c|c|c|c|c|c|c|c|c|c|c|c|c|c|}
\hline \multirow[b]{2}{*}{ IES } & \multirow{2}{*}{$\begin{array}{l}\mathrm{N}^{\mathrm{o}} \text { Total } \\
\text { de Prof. }\end{array}$} & \multicolumn{2}{|c|}{$\operatorname{Sexo}(\%)$} & \multicolumn{6}{|c|}{ Formação (\%) } & \multicolumn{5}{|c|}{ Mestrado } & \multicolumn{5}{|c|}{ Doutorado } & \multicolumn{3}{|c|}{ Tempo de Atuação no local (\%) } \\
\hline & & $\mathrm{F}$ & $\mathrm{M}$ & Far & Méd. & Bio & Quim & Outros & N.I. & RS & BR. & Ex & Total & N.I. & $\overline{\mathrm{RS}}$ & $\mathrm{BR}$ & Ext. & Total & $\overline{\text { N.I }}$ & +20 anos & $10-20$ anos & -10 anos \\
\hline UFRGS & 32 & 54 & 46 & 42 & 29 & 13 & 6 & 10 & & 25 & 2 & - & 27 & & 8 & 17 & 7 & 32 & & $16(50)$ & $9(28,1)$ & $7(21,8)$ \\
\hline UFSM & 11 & 55 & 45 & 46 & 9 & 27 & 18 & - & & 10 & 1 & - & 11 & & 6 & 5 & - & 11 & & - & $7(63,7)$ & $4(36,3)$ \\
\hline UFPEL & 13 & 31 & 69 & 8 & - & - & 23 & 31 & $38 *$ & 6 & 2 & - & 8 & $5 *$ & 3 & 2 & 1 & 6 & $5^{*}$ & $1(7,7)$ & $4(30,8)$ & $3(23)$ \\
\hline UFCSPA & 6 & 100 & - & 17 & - & 50 & 33 & & & 3 & 2 & - & 5 & & 3 & 2 & 1 & 6 & & - & $1(16,7)$ & $5(83,3)$ \\
\hline FURG & 4 & - & 100 & - & - & $50 *$ & - & 25 & $25^{*}$ & 2 & - & 1 & 3 & $1 *$ & 2 & - & - & 2 & $1 *$ & - & - & $3(75)$ \\
\hline
\end{tabular}

* Professores que não possuem currículo Lattes.

Evidencia-se que na UFRGS, dos 32 professores, 26 fizeram mestrado, dos quais 24 cursados em instituições sediadas no Rio Grande do Sul e 2 fora do estado. Na UFSM, todos os pesquisadores possuem mestrado, sendo 10 formados no estado e apenas 1 em outro estado. Dos 6 professores da UFCSPA, 5 são mestres, sendo 3 no RS e 2 fora.

Destaca-se que apenas 1 dos pesquisadores, entre todas as instituições que foram alvo desta investigação, realizou mestrado em universidade do exterior. Observa-se a inexistência de currículo Lattes para 6 professores, 5 e 1, respectivamente da UFPel e na FURG.

Analisando-se a formação em nível de doutorado, observa-se que na UFRGS, na UFSM e na UFCSPA todos os professores possuem esta titulação. Já na UFPel, dentre os 11 professores, há apenas 6 doutores, sendo que, dos outros, 5 não possuem currículo na plataforma Lattes, impossibilitando precisar seu nível de pós-graduação. Na FURG, dentre os 4 professores, pode-se observar que 2 fizeram doutorado e 1 deles não possui currículo Lattes.

Dentre os 65 professores que atuam nas cinco instituições, apenas 17 estão alocados nos respectivos departamentos/setores de Bioquímica há mais de 20 anos, sendo que praticamente a totalidade destes é da UFRGS. Isto pode ser explicado pela maior antiguidade do departamento de Bioquímica na UFRGS, manutenção de seus pesquisadores nessa atividade e criação mais recente de setores de Bioquímica na maioria das outras Instituições estudadas. Entre 10 e 20 anos de atuação, encontram-se 21 professores, chamando a atenção de que, neste grupo, está a maior proporção de professores da UFSM. Os outros 21 professores estão há menos de 10 anos em atividade no local e cargos atuais, distribuídos em todas as instituições em estudo.

Considerando-se o período compreendido entre 1999 e 2007, percebe-se que a maior produção científica veio da UFRGS, totalizando mais do que o dobro da soma das demais universidades, o que pode estar relacionado ao número de professores e ao fato do Programa de Pós-Graduação em nível de Mestrado e Doutorado nesta instituição ser o mais antigo. Na sequência, por número de artigos publicados, apresentam-se UFSM, FURG, UFPel e UFCSPA, conforme Tabela 2. A UFSM sobressai em sua produtividade tendo quintuplicado o número de artigos entre 1999 a 2007 e também possui Programa de Mestrado e Doutorado diretamente vinculado à área de Bioquímica.

Dos 984 artigos publicados pelos professores do Departamento de Bioquímica da UFRGS, 920 são indexados no Science Citation Index (SCI), que é a base de dados do Institute for Scientific Information (ISI), divulgado pelo Journal Citation Report (JCR). Na UFSM, das 371 publicações, apenas 16 não são indexados e na FURG dos 62 artigos, 41 são indexados. Já na UFPel e UFCSPA, a maioria dos artigos não é indexada. Estes dados podem refletir a vocação científica dos diferentes centros, levando à opção por publicações em periódicos indexados ou não, dependendo da visibilidade desejada.

Verifica-se que a produção científica no período do estudo apresenta picos. Entretanto, de 2004 até o final do estudo, evidencia-se que o número de artigos publicados é crescente em relação aos demais anos.

A UFRGS destaca-se pela orientação de pós-graduação. Totalizam-se 198 orientações de mestrado e 102 de doutorado no período estudado. Os anos de 2004 e 2005 demonstraram também o maior número, com 51/30 orientações, respectivamente. Atenta-se para a inexistência de programa de pós-graduação na UFCSPA onde professores de Bioquímica sejam orientadores. Nas outras instituições (UFPel e FURG), alguns dos professores de Bioquímica orientam em cursos da instituição existentes em áreas afins, o que pode explicar as poucas orientações identificadas. Muitos dos professores que atuam na UFSM foram formados pelos professores do Departamento de Bioquímica da UFRGS, talvez sendo esta a razão de um perfil parecido de produção e vocação científica entre as duas. Alguns dos professores da UFCSPA também foram orientados pelos professores da Bioquímica da UFRGS, mas como não possuem PPG têm dificuldade de publicar e, consequentemente, recebem menos fomento. Mas talvez o dado mais importante seja o fato de ser um grupo de professores com pouco tempo de atuação na instituição. De qualquer forma, o processo pelo qual o orientado foi formado pode ter sido importante na nucleação, por exemplo, em Santa Maria e, mais recentemente, na UFCSPA. Assim como os orientadores servem como modelo, os locais também podem contribuir nesta formação.

Estes resultados referidos até aqui foram reunidos como um conjunto que resume os dados de produtividade, no que se refere à publicação de artigos científicos e formação de recursos humanos (orientação de dissertações e teses). A partir destes, passamos a apresentar os dados individuais, na forma de gráficos, para dar maior visibilidade aos detalhes que nos parecem importante discriminar.

As Figuras 1a-c e 2a-b representam, na forma de histograma, a produção científica de cada um dos departamentos/setores de Bioquímica (discriminando trabalhos indexados e não indexados) e as orientações de dissertações e teses, de forma a se poder avaliar a relação existente entre produtividade e orientação, durante o período de 1999-2007. Isto nos indica que parece haver uma dependência

Tabela 2. Produção científica e formação de recursos humanos dos Departamentos/Setores no período de 1999-2007

\begin{tabular}{|c|c|c|c|c|c|c|c|c|c|c|c|c|c|c|c|c|c|c|c|c|c|c|c|c|c|c|c|c|c|c|c|}
\hline \multirow[t]{2}{*}{ Inst. } & \multirow{2}{*}{$\begin{array}{l}\text { Ano da Fundação } \\
\text { do Dep./Setor }\end{array}$} & \multirow{2}{*}{\multicolumn{2}{|c|}{ PPG }} & \multirow{2}{*}{$\begin{array}{l}\text { Produção do Período } \\
\text { (Indexado) }\end{array}$} & \multicolumn{9}{|c|}{ Produção no Período (99 a 07) } & \multicolumn{9}{|c|}{ Orientação de Mestrado (99 a 07) } & \multicolumn{9}{|c|}{ Orientação de Doutorado (99 a 07) } \\
\hline & & & & & 99 & 00 & 01 & 02 & $\mathbf{0 3}$ & 04 & 05 & 06 & 07 & 99 & 00 & 01 & 02 & 03 & 04 & 05 & 06 & 07 & 99 & 00 & 01 & 02 & $\mathbf{0 3}$ & 04 & 05 & 06 & 07 \\
\hline UFRGS & 1972 & 1977 & 771992 & $984(920)$ & 60 & 89 & 90 & 94 & 132 & 127 & 138 & 115 & 139 & 13 & 24 & 17 & 20 & 25 & 28 & 23 & 28 & 20 & 8 & 14 & 6 & 11 & 10 & 13 & 17 & 12 & 11 \\
\hline UFSM & 1998 & 1998 & 982003 & 371 (355) & 16 & 12 & 22 & 20 & 41 & 50 & 63 & 66 & 81 & 0 & 7 & 10 & 16 & 5 & 27 & 7 & 17 & 19 & - & - & - & - & 1 & 2 & 7 & 3 & 7 \\
\hline UFPel & 1969 & - & - & $38(9)$ & 1 & - & 2 & 4 & 1 & 3 & 12 & 5 & 10 & - & - & - & 1 & - & - & 2 & - & 2 & - & - & - & - & - & 1 & - & - & - \\
\hline UFCSPA & 1962 & - & - & $30(16)$ & 3 & 3 & 2 & 2 & 2 & 5 & 4 & 4 & 5 & - & - & - & - & - & - & - & - & - & - & - & - & - & - & - & - & - & - \\
\hline FURG & 1966 & - & - & $62(41)$ & - & - & - & 3 & 12 & 8 & 7 & 19 & 13 & - & - & - & - & 1 & 1 & 3 & 2 & 1 & - & - & - & - & - & - & - & - & - \\
\hline
\end{tabular}


dos alunos (orientandos) no aumento da produção científica, conforme citado na Introdução. ${ }^{1,22,25}$ Nas Figuras 1b-c e 2a-b os inserts representam os mesmos dados apresentados, mas utilizando a escala usada para a UFRGS 1a.

A produção científica e as informações sobre orientação de Mestrado e de Doutorado foram discriminadas ano a ano durante o período a que se refere o estudo (1999-2007), para cada uma das instituições - Figuras $1 \mathrm{Sa}-\mathrm{e}, 2 \mathrm{Sa}$-d e 3Sa-c, do material suplementar.

A Figura 1Sa, material suplementar, mostra que o Departamento de Bioquímica da UFRGS teve uma produtividade científica crescente, duplicando de 1999 a 2007. Este aumento de produção se repete na UFSM, mas com muito maior intensidade, praticamente

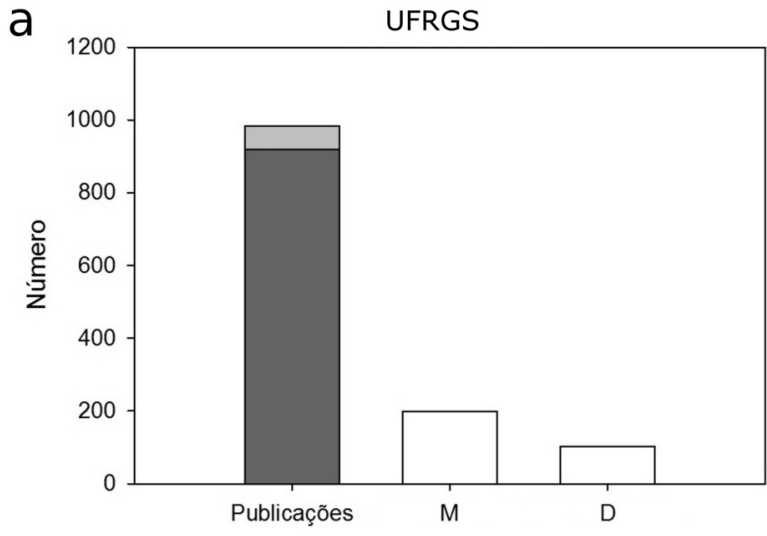

b

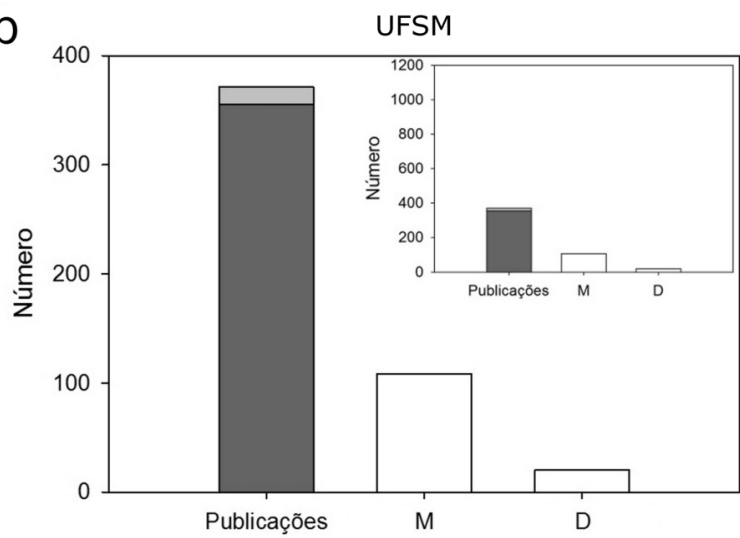

C

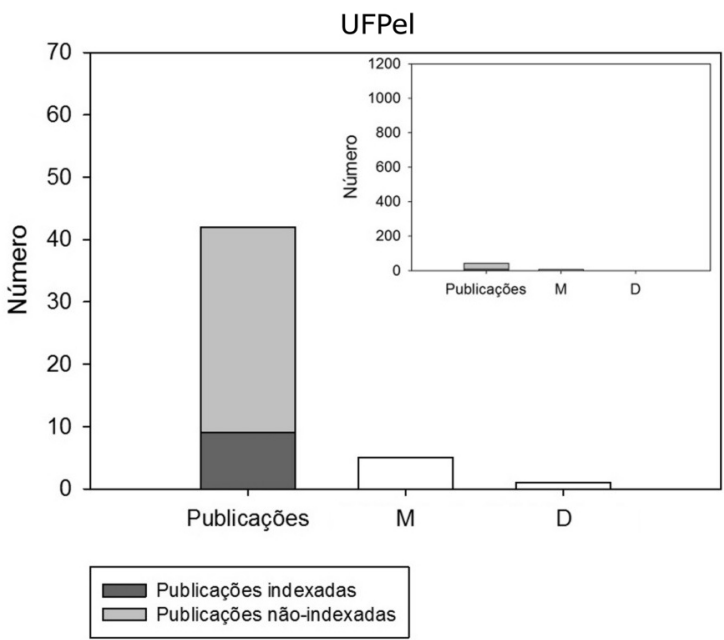

Figura 1. Publicações de artigos (indexados e não indexados) e orientação de Dissertações de Mestrado (M) e Teses de Doutorado (D) no período de 1999-2007: a-UFRGS; b-UFSM; c-UFPel quadruplicando ao longo do período estudado - Figura 1Sb.

Em relação às outras instituições, observamos um índice de produção científica menor, com alguns picos, sendo que na FURG só há registro de produção a partir de 2002 - Figura 1Sc-e, material suplementar.

Quanto à orientação de Mestrado, observamos que na UFRGS a orientação de dissertações é basicamente acompanhada pela produção de artigos (Figura 2Sa, material suplementar). Na UFSM a orientação de Mestrado iniciou em 2000 e triplicou neste período (Figura 2Sb). Na UFPel e na FURG houve um número muito pequeno de orientações de Mestrado, (Figuras 2Sc-d). Não houve nenhuma orientação de Mestrado pelos professores/pesquisadores na UFCMPA.

No período analisado também o número de Teses de Doutorado orientadas na UFRGS acompanhou a produção científica dos professores (Figura 3Sa, material suplementar).

Quanto à orientação de Doutorado na UFSM, os dados são compatíveis com o início recente desta atividade em 2003 (Figura 3Sb, material suplementar). Aliás, provavelmente este período corresponde àquele de importante crescimento científico que este grupo teve, pelas características de ser formado por vários pesquisadores jovens incorporados pela instituição na última década.

Nas outras instituições a relação da produção com orientação é difícil, pois, como já citado, não há Programa de Pós-Graduação diretamente vinculado ao setor, havendo alguma orientação ligada a Programas em interação com outros setores. Apenas na UFPel há registro de uma orientação em 2004 (Figura 3Sc, material suplementar).
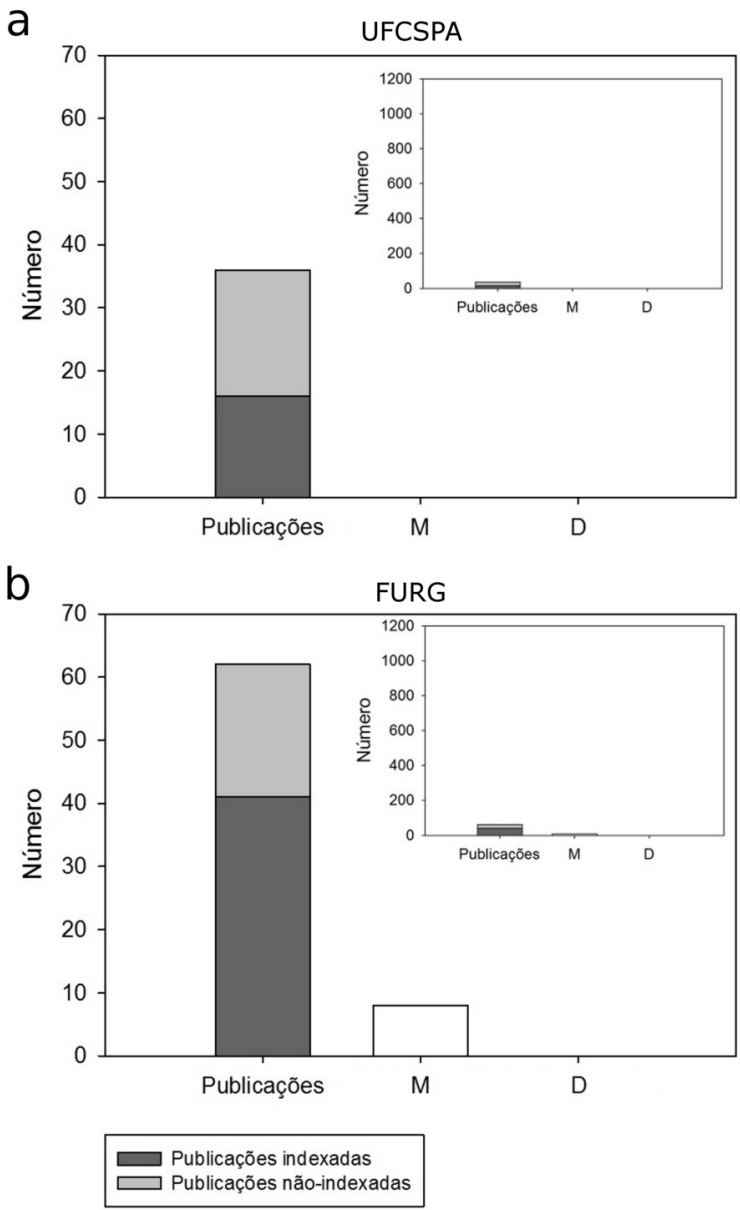

Figura 2. Publicações de artigos (indexados e não indexados) e orientação de Dissertações de Mestrado (M) e Teses de Doutorado (D) no período de 1999-2007: $a$ - UFCSPA; $b$ - FURG 
Tabela 3. Auxílios financeiros da Fundação de Amparo à Pesquisa do Estado do Rio Grande do Sul (FAPERGS), na forma de bolsas e auxílios individuais e institucionais (1999 a 2007)

\begin{tabular}{|c|c|c|c|c|c|c|c|c|c|c|c|c|}
\hline \multirow[b]{3}{*}{ Inst. } & \multicolumn{4}{|c|}{ Bolsas } & \multicolumn{6}{|c|}{ Auxílios Individuais } & \multicolumn{2}{|c|}{ Auxílios Institucionais } \\
\hline & \multicolumn{2}{|c|}{$\mathrm{BIC}$} & \multicolumn{2}{|c|}{ OUTRAS } & \multicolumn{3}{|c|}{$\mathrm{ADE}$} & \multicolumn{3}{|c|}{ OUTROS } & \multirow[t]{2}{*}{$\mathrm{R} \$$} & \multirow{2}{*}{$\begin{array}{c}\mathrm{N} \mathrm{de} \\
\text { auxílios }\end{array}$} \\
\hline & $\begin{array}{c}\mathrm{N} \text { de } \\
\text { auxílios }\end{array}$ & $\begin{array}{c}\text { Prof. Cont. } \\
(\%)\end{array}$ & $\begin{array}{c}\mathrm{N} \text { de } \\
\text { auxílios }\end{array}$ & $\begin{array}{c}\text { Prof. Cont. } \\
(\%)\end{array}$ & $\mathrm{R} \$$ & $\begin{array}{c}\mathrm{N} \text { de } \\
\text { auxílios }\end{array}$ & $\begin{array}{c}\mathrm{N} \text { de prof } \\
(\%)\end{array}$ & $\mathrm{R} \$$ & $\mathrm{~N}$ de auxílios & $\begin{array}{c}\mathrm{N} \text { de prof } \\
(\%)\end{array}$ & & \\
\hline UFRGS & 89 & $26(81,2)$ & 9 & $7(22,6)$ & 118.000 & 15 & $14(45,2)$ & $183.924,27$ & 21 & $14(45,2)$ & $688.825,22$ & 16 \\
\hline UFSM & 26 & $8(72,8)$ & 4 & $2(18,2)$ & 93.000 & 07 & $05(45,5)$ & $127.965,00$ & 12 & $7(63,6)$ & $4.000,00$ & 2 \\
\hline UFPel & 01 & $1(7,7)$ & - & - & - & - & - & $10.400,00$ & 02 & $02(15,4)$ & - & - \\
\hline UFCSPA & - & & & & & & & $7.498,00$ & 1 & $1(20)$ & - & - \\
\hline FURG & 03 & $02(50)$ & - & - & - & - & - & $60.762,00$ & 5 & $2(50)$ & $27.370,00$ & 01 \\
\hline
\end{tabular}

BIC: Bolsa de Iniciação Científica; OUTRAS BOLSAS correspondem a: Bolsa Professor Visitante (BPV) e Bolsa Recém-Doutor (BRD); Programa de Apoio ao Desenvolvimento Científico-Tecnológico-ArtísticoCultural do Estado do Rio Grande do Sul (ADE); OUTROS AUXÍLIOS INDIVIDUAIS correspondem a: Auxílio Participação Evento Internacional (APEI); Auxílio Participação Evento Nacional (APEN); Auxílio Organização de Eventos (AOE); Auxílio Professor Visitante (APV). Os institucionais referem-se a: Programa para Núcleos de Excelência (PRONEX); Programa de Apoio ao Desenvolvimento Científico-Tecnológico Regional no Estado do Rio Grande do Sul (PROCOREDES); Programa de Pesquisa para o Sistema Único de Saúde (PPSUS).

A FAPERGS - Fundação de Amparo à Pesquisa do Estado do Rio Grande do Sul - possui programas de fomento à Iniciação Científica e outros de formação de recursos humanos, além de auxílios individuais e institucionais à pesquisa. Os valores referentes a essas modalidades de fomento concedido às cinco universidades estudadas estão apresentados na Tabela 3.

Observa-se que a UFRGS recebeu 89 Bolsas de Iniciação Científica (BIC) que foram repassadas a 26 professores, representando $81,3 \%$ do quadro do Departamento. Percentual aproximado verifica-se na UFSM, onde 8 professores $(72,8 \%$ ) foram contemplados com 26 bolsas. O menor percentual ocorreu na UFPel, que recebeu apenas 1 BIC, sendo que a UFCSPA não recebeu nenhuma bolsa.

Em referência a outras modalidades de bolsas, como Bolsa Professor Visitante (BPV) e Bolsa Recém-Doutor (BRD), destaca-se que somente UFRGS e UFSM foram contempladas, sendo a primeira com 9 e a outra com 4, atingindo em torno de $20 \%$ dos professores. Analisando-se os auxílios individuais da categoria Programa de Apoio ao Desenvolvimento Científico-Tecnológico-Artístico-Cultural do Estado do Rio Grande do Sul (ADE), estas duas instituições também foram às únicas contempladas, sendo que a UFRGS recebeu 15 auxílios destinados a 14 professores. De outra parte, a UFSM recebeu 7 auxílios para 5 professores. Nos dois casos, $45 \%$ dos professores receberam este auxílio, que é destinado a financiamento de projetos individuais de pesquisa.

Com relação aos demais auxílios individuais, como Auxílio Participação Evento Internacional (APEI); Auxílio Participação Evento Nacional (APEN); Auxílio Organização de Eventos (AOE); Auxílio Professor Visitante (APV), todas as Instituições foram financiadas, mas se destacam a UFRGS, UFSM e FURG como as principais contempladas com 21, 12 e 5 auxílios, respectivamente, atingindo em torno da metade dos professores.

Na modalidade auxílios institucionais, integrada por Programa para Núcleos de Excelência (PRONEX); Programa de Apoio ao Desenvolvimento Científico-Tecnológico Regional no Estado do Rio Grande do Sul (PROCOREDES); Programa de Pesquisa para o Sistema Único de Saúde (PPSUS), entre outros, observa-se que a UFRGS se sobressai tendo recebido 16 dos 19 concedidos, totalizando um montante superior a R \$ 680 mil. Observa-se que a UFSM recebeu auxílio institucional em valor menor que aquele auxílio recebido pela FURG.

Buscou-se, com este trabalho de pesquisa, mapear as áreas que são desprovidas de fomento, podendo assim apontar às agências os setores mais carentes de recursos.

Observou-se que o critério de distribuição de verbas da FAPERGS é similar ao das agências de fomento federais como CAPES, CNPq e FINEP. A Fundação concedeu mais apoio, por meio de seus programas, aos departamentos já consolidados, que mais produzem e publicam. Evidenciou-se que onde a produção é menor, a alocação é mais reduzida. Em linhas gerais, os investimentos da FAPERGS no período de abrangência deste estudo, podem ser considerados, como um todo, relevantes.

Os investimentos foram destinados, em sua maioria, ao Departamento de Bioquímica da UFRGS, considerado centro de excelência no país (com Programa de Pós-Graduação nível 7 pela CAPES), que possui o maior número de pesquisadores pós-graduados, a maior quantidade de publicações em revistas indexadas e orientações de mestrado e doutorado, permitindo formação contínua de recursos humanos qualificados. A UFRGS conta com grande número de auxílios institucionais.

O Departamento de Bioquímica da UFSM praticamente não recebeu, neste período, auxílios institucionais. Contudo, é equivalente à UFRGS no recebimento de auxílios individuais e possui alta produção em revistas indexadas. A quantidade de orientação de mestrado e doutorado é crescente. A maioria dos pesquisadores de seu quadro foi orientada por professores da bioquímica da UFRGS, fator que pode ter conduzido seu departamento à segunda posição entre as universidades estudadas em número de publicações, orientação e captação de recursos.

Na UFPel a iniciação científica apresenta-se bastante defasada em relação às duas universidades acima citadas. As orientações de mestrado e doutorado são raras. Percebeu-se que os auxílios institucionais e auxílios individuais são raros. Entretanto, há importante presença de artigos científicos em revistas não indexadas, o que pode significar que estas são publicações relevantes para o enfoque de suas pesquisas e para os seus objetivos científicos.

FURG e UFCSPA têm perfil semelhante à UFPel no que tange à iniciação científica, auxílios institucionais e auxílios individuais. Suas áreas de Bioquímica possuem ainda baixo índice de publicações.

Considera-se que a FAPERGS deve reeditar ou ampliar programas de apoio à formação de RH, como BIC, bolsa emergencial de mestrado e doutorado, e apoio à pesquisa, a exemplo de recém-mestre e recém-doutor que estimulem estas interações. Evidencia-se também a necessidade de ampliação do apoio a maior número de professores dos setores menos produtivos, por intermédio de seus programas de fomento, além de manter aqueles aos Departamentos de Bioquímica melhor estruturados (UFRGS e UFSM), pois a alta e relevante produção justificam a demanda.

Após refletir acerca dos dados coletados e apresentados, aponta-se a necessidade de interação entre os centros de forma a aumentar e intensificar interrelacionamentos e parcerias entre os departamentos/ setores considerados mais e menos estruturados, aumentando assim o número de pesquisadores e orientadores entre os professores de Bioquímica. A interação resultante entre UFRGS e UFSM parece um exemplo, provavelmente oriunda da incorporação de professores/ pesquisadores na UFSM formados na UFRGS. Este exemplo pode nortear interações entre todas as Instituições. 


\section{CONCLUSÕES}

Pode-se concluir que há necessidade de ampliar as interações entre as diferentes Universidades Públicas Federais do Rio Grande do Sul. Desta maneira, poderia se contribuir para que os departamentos/ setores de bioquímica menos estruturados, como é o caso de UFPel, FURG e UFCSPA, pudessem estruturar programas de pós-graduação em parceria com UFRGS e UFSM, que apresentam os melhores indicadores em produção científica. Esta aproximação institucional, também, poderá propiciar acesso a auxílios e bolsas para formação de recursos humanos e fomento à pesquisa.

Evidenciou-se a necessidade da FAPERGS reeditar programas para a formação de RH, bem como ampliar o apoio financeiro aos departamentos/setores de bioquímica de UFPEL, FURG e UFCSPA. Aponta-se ainda que UFRGS e UFSM, mesmo já sendo detentoras da maior parte dos recursos concedidos, em razão de sua relevante produtividade, demandam um crescimento contínuo de investimentos públicos.

\section{MATERIAL SUPLEMENTAR}

No material suplementar, disponível gratuitamente em http:// quimicanova.sbq.org.br na forma de arquivo PDF, são mostrados os artigos publicados ano a ano no período de 1999 a 2007 - Figura 1S (a-e), assim como Dissertações de Mestrado ano a ano no período de 1999 a 2007 - Figura 2S (a-d) e Teses de Doutorado ano a ano no período de 1999 a 2007 -Figura 3S (a-c).

\section{AGRADECIMENTOS}

O presente trabalho foi realizado com o auxílio da CAPES (Coordenação de Aperfeiçoamento de Pessoal de Nível Superior) e do INCT (Instituto Nacional de Ciência e Tecnologia em Citotoxicidade e Neuroproteção).

\section{REFERÊNCIAS}

1. Glanzel, W.; Leta, J.; Thus, B.; Scientometrics 2006, 67, 67.

2. De Meis, L.; Longo. P.; Biochem Educ. 1990, 18, 182.

3. Leta, J.; Glänzel, W.; Thus, B.; Scientometrics 2006, 67, 87.

4. Veloso, A.; Lannes, D.; De Meis, L.; Scientometrics 2004, 61, 207

5. Fonseca, L.; Velloso, S.; Wofchuk, S.; De Meis, L.; Scientometrics 1998, $41,299$.

6. Merton, R. K.; Social theory and social structure, Free Press: New York, 1957.
7. Okubo, Y.; Science, Technology and Industry Working Papers 1997, doi:10.1787/208277770603.

8. Price, D.; Little science, big science, Columbia University Press: New York, 1963, edição 119.

9. Cavalcanti, I. G. M.; Gómez, M. N. G.; Araujo, I. M.; Ribeiro, R. B. C.; Rosário, J. S.; Silva, A. P.; Fernandéz, G. R.; Resumos do Seminário Nacional de Bibliotecas Universitárias, Florianópolis, Brasil, 2000.

10. Araujo, K. M.; Mourão, P. A. S.; Leta, J.; Braz. J. Med. Biol. Res. 2005, 38,1285 .

11. Leta, J.; Chaimovich, H.; Scienciometrics 2002, 53, 325.

12. Leta, J.; Scientometrics 2002, 53, 325.

13. King, J.; Journal of Information Science 1987, 13, 261.

14. Gracelli, A.; Castro, C.; Cien. Cult. 1985, 37, 188.

15. http://www.capes.gov.br/images/stories/download/bolsas/INF1E295.pdf, acessada em Junho 2006.

16. http://www.capes.gov.br/scripts/p.prog.ide, acessada em Junho 2006.

17. Meneghini, R.; Fonseca, L.; Cien. Cult. 1990, 42, 629.

18. Meneghini, R.; Scientometrics 1996, 35, 267.

19. Smit, J. W.; Informação \& Sociedade 1999, 9, 385.

20. Castro, C.; Cien. Cult. 1985, 37, 7.

21. Rocha Netto, I.; Educação Brasileira 1988, 10, 17.

22. Fonseca, L.; Velloso, S.; Wofchuk, S.; De Meis, L.; Scientometrics 1997, $39,159$.

23. Harmon, L. R. Em Scientific creativity; Its recognition and developmen; Taylor, C. W.; Barron, F., eds.; John Wiley \& Sons: New York, 1963.

24. Sonnert, G.; Social Studies of Science 1995, 25, 35.

25. De Meis, L.; Leta, J.; O Perfil da Ciência Brasileira, Ed. UFRJ: Rio de Janeiro, 1996

26. Leta, J.; Lannes, D.; De Meis, L.; Scientometrics 1998, 41, 313.

27. Braga, M.; Azevedo, S.; Quim. Nova 2002, 25, 866.

28. Prado, J. L. Em A História das Ciências no Brasil; Ferri, M. G.; Motoyama, S., eds.; EDUSP: São Paulo, 1979, cap. 5.

29. Martins, T. Em As Ciências no Brasil; Azevedo, F., ed.; $2^{\text {a }}$ ed., Ed. UFRJ: Rio de Janeiro, 1994, cap. 11.

30. Prado, J. L.; Cien. Cult. 1978, 30, 549.

31. Schwartzman, S.; Formação da Comunidade Científica no Brasil, Editora Nacional: São Paulo, 1979, caps. 7 e 9.

32. http://conteudoweb.capes.gov.br/conteudoweb/ProjetoRelacaoCursosS ervlet $? \mathrm{acao}=$ pesquisarArea $\&$ codigoGrandeArea $=20000006 \&$ descricao GrandeArea=CI\%CANCIAS+BIOL\%D3GICAS+, acessada em Março 2009.

33. Gil, A.; Métodos e técnicas de pesquisa social, $5^{\text {a }}$ ed., falta editora: São Paulo, 1999. 


\section{PRODUÇÃO CIENTÍFICA E FORMAÇÃO DE RECURSOS HUMANOS NA ÁREA DE BIOQUÍMICA EM INSTITUIÇÕES FEDERAIS DO RIO GRANDE DO SUL: FOMENTO ESTADUAL}

Luciana Calabró Berti*, Diogo L. Oliveira, Diogo O. Souza e Susana T. Wofchuk

Departamento de Bioquímica, Instituto de Ciências Básicas da Saúde, Universidade Federal do Rio Grande do Sul, Rua Ramiro Barcellos, 2600 - Anexo, 90035-003 Porto Alegre, RS - Brasil
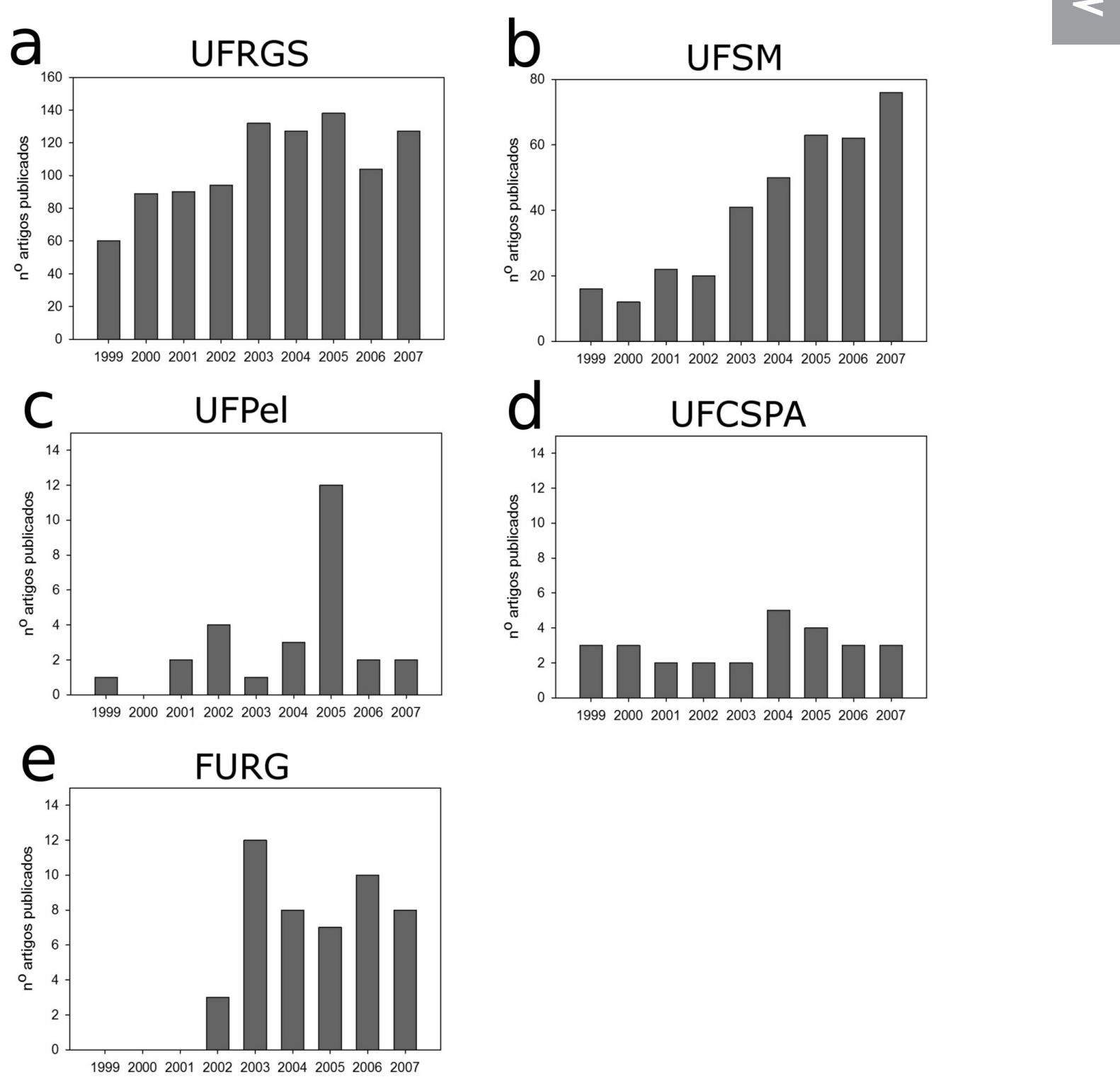

Figura 1S. Artigos publicados ano a ano no período de 1999 a 2007 na UFRGS (a), na UFSM (b), na UFPel (c), na UFCSPA (d) e na FURG (e) 

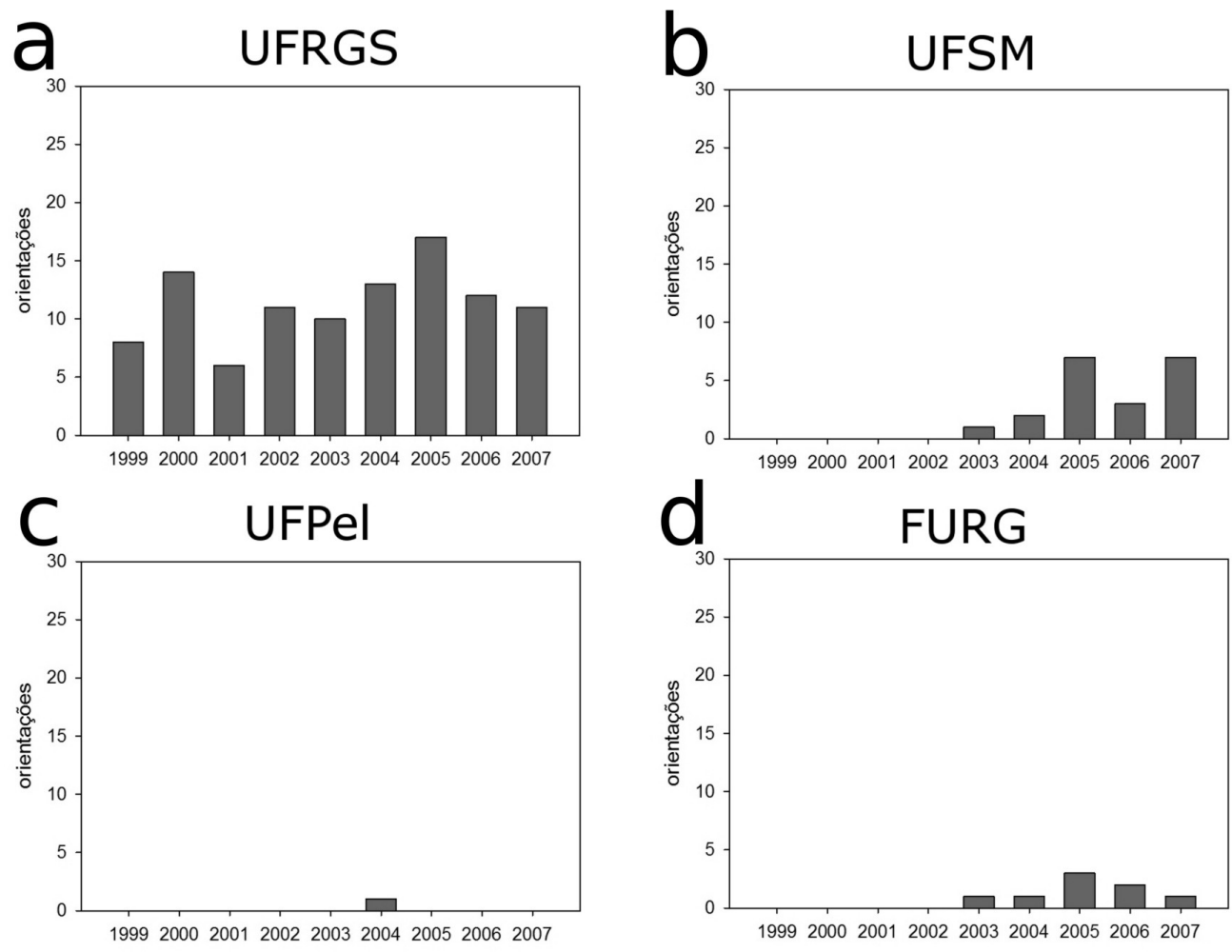

Figura 2S. Dissertações de Mestrado orientadas ano a ano no período de 1999 a 2007 na UFRGS (a), na UFSM (b), na UFPel (c) e na FURG (d)
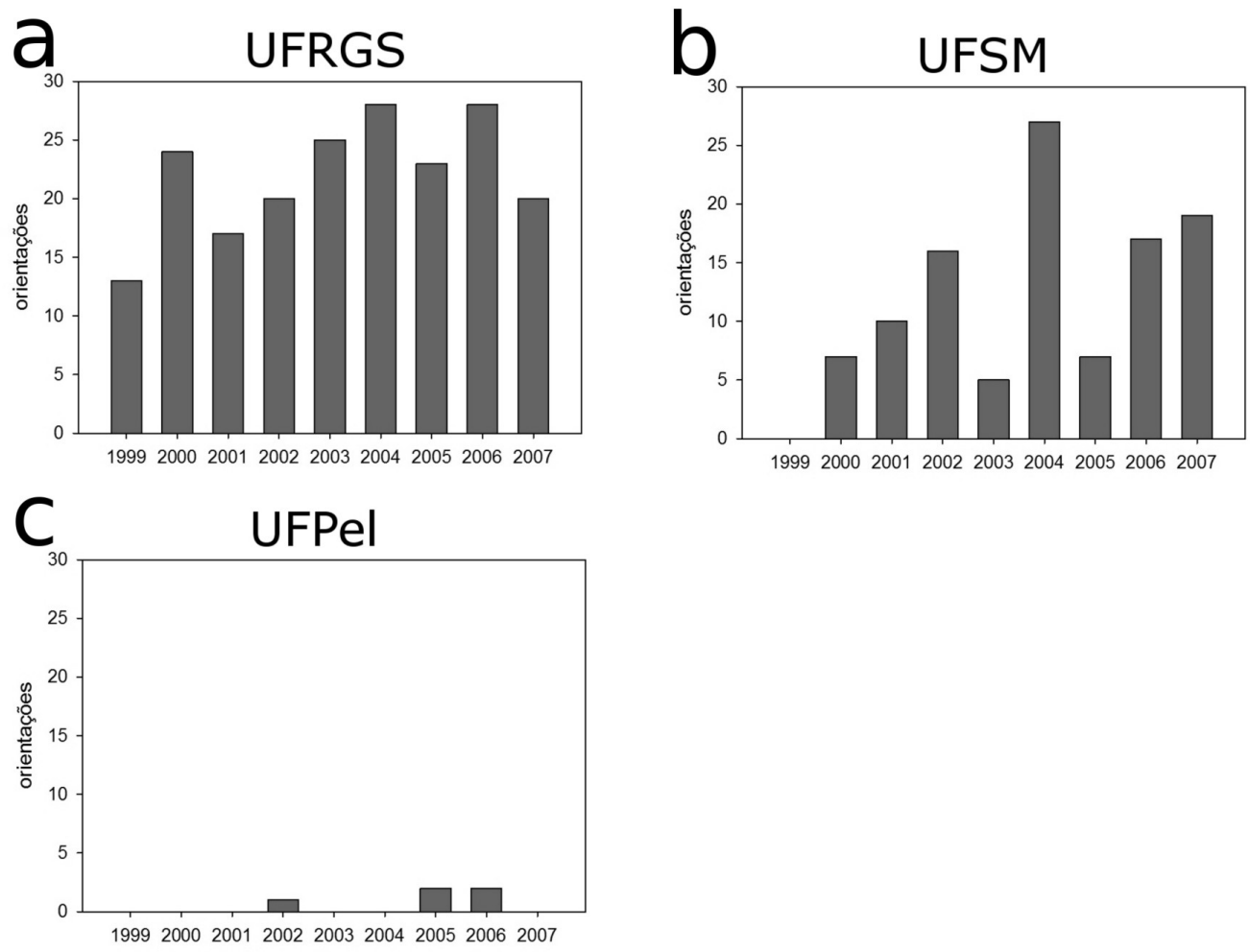

Figura 3S. Teses de Doutorado orientadas ano a ano no período de 1999 a 2007 na UFRGS (a), na UFSM (b) e na UFPel (c) 